\begin{tabular}{|c|c|}
\hline Title & Optical Observation of Quasi periodic Heisenberg A ntiferromagnets in Two Dimensions \\
\hline Author(s) & Inoue, Takashi; Y amamoto, Shoji \\
\hline Citation & $\begin{array}{l}\text { Physica status solidi B-basic solid state phy sics, 257(11), } 2000118 \\
\text { https://doi.org/10.1002/pssb.202000118 }\end{array}$ \\
\hline Issue Date & $2020-11$ \\
\hline Doc URL & http://hdl .handle.net/2115/83127 \\
\hline Rights & $\begin{array}{l}\text { This is the peer reviewed version of the following article:Phy sica status solidi B-basic solid state physics } 2000118 \\
\text { July } 2020,2000118 \text { which has been published in final form at } \\
\text { https://onlinelibrary.wiley .com/doi full/10.1002/pssb.202000118. This article may be used for non-commercial } \\
\text { purposes in accordance with Wiley Terms and Conditions for U se of Self-A rchived V ersions. }\end{array}$ \\
\hline Tyре & article \\
\hline File Information & Phys. Status Solidi B-Basic Solid State Phys. 2000118.pdf \\
\hline
\end{tabular}

Instructions for use 


\title{
Optical observation of quasiperiodic Heisenberg antiferromagnets in two dimensions
}

\author{
Takashi Inoue and Shoji Yamamoto* \\ Department of Physics, Hokkaido university, Sapporo 060-0810, Japan
}

Key words: Quasicrystal, Heisenberg antiferromagnet, Raman scattering, Spin-wave theory

${ }^{*}$ Corresponding author: e-mail yamamoto@phys.sci.hokudai.ac.jp

We make a spin-wave analysis of magnetic Raman scattering in an antiferromagnetic Heisenberg model on the two-dimensional Penrose lattice of $\mathrm{C}_{5 \mathrm{v}}$ point symmetry. Following the Shastry-Shraiman perturbation scheme for a strongly correlated Hubbard model, we go so far as to obtain the fourth-order effective Raman operators. Within the second-order mechanism, there is one and only Raman active mode of $E_{2}$ symmetry, yielding a spectral weight independent of light polarization. Taking account of the fourth-order scatterings as well activates $A_{1}$ and $A_{2}$ as well as $E_{2}$ modes and therefore results in polarization-dependent Raman spectra. With the use of linearly and circularly polarized lights, we can separately extract all the symmetry species from observations. Though the linear spin-wave theory, i.e. the harmonic oscillator approximation, is far from quantitative, the $A_{2}$ and $E_{2}$ Raman intensities are well describable with two magnon interactions. The Raman-active $A_{1}$ mode owes much to higher order magnon-magnon interactions as well.

\section{Introduction}

Since the discovery of quasicrystal [1], quasiperiodic systems have been of much interest. Quasicrystals are characterized by quasiperiodicity, which is a coexistence of long-range order without translational symmetry and crystallographically forbidden rotational symmetry. These expect that the physical properties of quasiperiodic systems are quite different from both periodic and amorphous systems. The Penrose lattice is one of the most popular two-dimensional quasicrystals. On this lattice, the tightbinding model of noninteracting electrons has been studied. It exhibits many interesting features, such as confined states $[2,3]$ characterized by thermodynamically degenerate states with strictly localized and self-similar wave functions, and multifractal spectrum [4]. Recently, quantum critical behavior has been observed in the quasicrystal $\mathrm{Au}_{51} \mathrm{Al}_{34} \mathrm{Yb}_{15}$ [5]. In this compound, the $4 f$ electrons of $\mathrm{Yb}$ are strongly correlated. Investigating the interplay between quasiperiodicity and strong correlation is a big issue. In the quasiperiodic systems, strongly correlated electron models have been studied such as Hubbard model [6], Ising model for classical spins [7], and Heisenberg model for quantum spins $[8,9]$.
One of the important probes of antiferromagnets is magnetic Raman scattering. It is inelastic photon scattering mediated by magnetic excitations. Loudon and Fleury established a standard framework for the two-magnon Raman scattering [10]. For instance, it was used to estimate the exchange interaction constant of the high- $T_{c}$ superconductor $\mathrm{La}_{2} \mathrm{CuO}_{4}$ [11]. The insulating phase of layered cuprates can be well accounted for quasi-two-dimensional Heisenberg antiferromagnets on the square lattice. The magnetic Raman spectra at absolute zero temperature have been calculated by spin-wave theory [12-14], exact diagonalization [14], and quantum Monte Carlo methods [14]. Magnetic Raman spectra have also been computed for other systems such as the triangular [15] and Kagome [16] lattices. The polarization dependence of the magnetic Raman intensity depends on the geometry of the lattice and the symmetry of the ground state. It provides useful information on magnetic excitation.

Microscopic description of the magnetic Raman scattering was given by Shastry and Shraiman $[17,18]$. In this formulation, the Loudon-Fleury mechanism is obtained in second-order perturbation theory. Magnetic Raman scattering beyond the Loudon-Fleury mechanism is obtained in 
higher-order perturbation. It includes additional magnetic excitations, such as the spin-chirality terms $\boldsymbol{S}_{i} \cdot\left(\boldsymbol{S}_{j} \times \boldsymbol{S}_{k}\right)$ and/or the ring-exchange terms $\left(\boldsymbol{S}_{i} \cdot \boldsymbol{S}_{j}\right)\left(\boldsymbol{S}_{k} \cdot \boldsymbol{S}_{l}\right)[19$, 20]. We present the Raman intensity profile of the Penrose lattice Heisenberg antiferromagnet within and beyond the Loudon-Fleury mechanism by spin-wave theory. Beyond the Loudon-Fleury mechanism does the $A_{2}$ mode becomes Raman active, with its dynamic spin-chirality fluctuations owing much to the quasiperiodic lattice structure. A comparison with exact diagonalization reveals the effects of magnon-magnon interactions.

\section{Model}

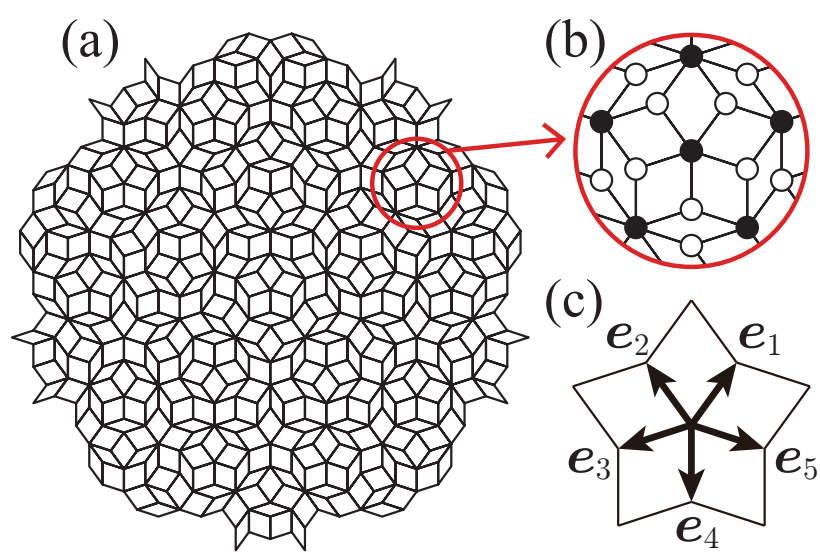

Figure 1 Finite patch of the two-dimensional Penrose lattice with fivefold rotational symmetry (a) and enlarged view of the circled area (b). Closed and open circles represent vertices that belong to sublattice $\mathrm{A}$ and $\mathrm{B}$, respectively. Primitive lattice vectors of the Penrose lattice $\boldsymbol{e}_{1}, \cdots, \boldsymbol{e}_{5}$ (c) are projection of the fivedimensional canonical basis vectors, and they satisfy $\boldsymbol{e}_{1}+\boldsymbol{e}_{2}+$ $e_{3}+e_{4}+e_{5}=\mathbf{0}$

\subsection{Penrose lattice}

Figure 1 shows a finite cluster of the Penrose lattice. It is constructed from two prototiles: thin (acute angle $\pi / 5$ ) and thick (acute angle $2 \pi / 5$ ) rhombuses with equal edge length. Since the lattice consists of even-numbersided polygons, the Penrose lattice is bipartite. The twodimensional Penrose lattice is obtained by projecting a five-dimensional hypercubic lattice onto an irrational tilted plane [8]. The Penrose lattice holds four independent primitive lattice vectors. Due to the quasiperiodicity, the rank of the Penrose lattice $r=4$ is greater than the lattice dimension $d=2$. In this study, we consider open-boundary clusters of the Penrose lattice with fivefold rotational symmetry.

\subsection{Hamiltonian}

We consider the so-called vertex model, where spins are located at the vertices of the Penrose rhombus tiling. We consider the nearest-neighbor antiferromagnetic Heisen- berg model:

$$
H=J \sum_{\langle i, j\rangle} \boldsymbol{S}_{i} \cdot \boldsymbol{S}_{j} \quad(J>0)
$$

where $\boldsymbol{S}_{i}$ is a spin-1/2 operator at site $i$, and $\langle i, j\rangle$ are pairs of linked vertices of the Penrose lattice.

\subsection{Spin-wave theory}

We divide the Penrose lattice into two sublattices A and B consisting of $N_{\mathrm{A}}$ and $N_{\mathrm{B}}$ sites, respectively. In the classical ground state of the non-frustrated system, all A sublattice spins point in one direction and the B sublattice spins point in the opposite direction. We introduce bosonic operators by using the Holstein-Primakoff transformation:

$$
\begin{aligned}
S_{i}^{z} & =S-a_{i}^{\dagger} a_{i} \\
S_{i}^{+} & =\left(2 S-a_{i}^{\dagger} a_{i}\right)^{\frac{1}{2}} a_{i} \\
S_{i}^{-} & =a_{i}^{\dagger}\left(2 S-a_{i}^{\dagger} a_{i}\right)^{\frac{1}{2}}
\end{aligned}
$$

for $i \in \mathrm{A}$, and

$$
\begin{aligned}
S_{j}^{z} & =-S+b_{j}^{\dagger} b_{j} \\
S_{j}^{+} & =b_{j}^{\dagger}\left(2 S-b_{j}^{\dagger} b_{j}\right)^{\frac{1}{2}} \\
S_{j}^{-} & =\left(2 S-b_{j}^{\dagger} b_{j}\right)^{\frac{1}{2}} b_{j}
\end{aligned}
$$

for $j \in \mathrm{B}$. Expanding the square roots of $1 / S$, and keeping terms of up to $O\left(S^{0}\right)$, spin-wave Hamiltonian is written as

$$
\begin{aligned}
H_{\mathrm{SW}}= & J \sum_{\langle i, j\rangle}\left[-S^{2}+S\left(a_{i}^{\dagger} a_{i}+b_{j}^{\dagger} b_{j}+a_{i} b_{j}+a_{i}^{\dagger} b_{j}^{\dagger}\right)\right. \\
& \left.-\left\{a_{i}^{\dagger} a_{i} b_{j}^{\dagger} b_{j}+\frac{1}{4}\left(a_{i}^{\dagger} a_{i} a_{i} b_{j}+a_{i}^{\dagger} b_{j}^{\dagger} b_{j}^{\dagger} b_{j}+\text { H.c. }\right)\right\}\right]
\end{aligned}
$$

We apply the Wick decomposition to the $O\left(S^{0}\right)$ terms in Equation (4),

$$
\begin{aligned}
a_{i}^{\dagger} a_{i} b_{j}^{\dagger} b_{j} \rightarrow & \left\langle a_{i}^{\dagger} a_{i}\right\rangle b_{j}^{\dagger} b_{j}+\left\langle b_{j}^{\dagger} b_{j}\right\rangle a_{i}^{\dagger} a_{i}-\left\langle a_{i}^{\dagger} a_{i}\right\rangle\left\langle b_{j}^{\dagger} b_{j}\right\rangle \\
& +\left\langle a_{i}^{\dagger} b_{j}^{\dagger}\right\rangle a_{i} b_{j}+\left\langle a_{i} b_{j}\right\rangle a_{i}^{\dagger} b_{j}^{\dagger}-\left\langle a_{i}^{\dagger} b_{j}^{\dagger}\right\rangle\left\langle a_{i} b_{j}\right\rangle \\
a_{i}^{\dagger} a_{i} a_{i} b_{j} \rightarrow & 2\left(\left\langle a_{i}^{\dagger} a_{i}\right\rangle a_{i} b_{j}+\left\langle a_{i} b_{j}\right\rangle a_{i}^{\dagger} a_{i}-\left\langle a_{i}^{\dagger} a_{i}\right\rangle\left\langle a_{i} b_{j}\right\rangle\right) \\
a_{i}^{\dagger} b_{j}^{\dagger} b_{j}^{\dagger} b_{j} \rightarrow & 2\left(\left\langle a_{i}^{\dagger} b_{j}^{\dagger}\right\rangle b_{j}^{\dagger} b_{j}+\left\langle b_{j}^{\dagger} b_{j}\right\rangle a_{i}^{\dagger} b_{j}^{\dagger}-\left\langle a_{i}^{\dagger} b_{j}^{\dagger}\right\rangle\left\langle b_{j}^{\dagger} b_{j}\right\rangle\right) \\
a_{i}^{\dagger} a_{i}^{\dagger} a_{i} b_{j}^{\dagger} \rightarrow & 2\left(\left\langle a_{i}^{\dagger} a_{i}\right\rangle a_{i}^{\dagger} b_{j}^{\dagger}+\left\langle a_{i}^{\dagger} b_{j}^{\dagger}\right\rangle a_{i}^{\dagger} a_{i}-\left\langle a_{i}^{\dagger} a_{i}\right\rangle\left\langle a_{i}^{\dagger} b_{j}^{\dagger}\right\rangle\right) \\
a_{i} b_{j}^{\dagger} b_{j} b_{j} \rightarrow & 2\left(\left\langle a_{i} b_{j}\right\rangle b_{j}^{\dagger} b_{j}+\left\langle b_{j}^{\dagger} b_{j}\right\rangle a_{i} b_{j}-\left\langle a_{i} b_{j}\right\rangle\left\langle b_{j}^{\dagger} b_{j}\right\rangle\right)
\end{aligned}
$$

where $\langle\cdots\rangle$ denotes a quantum average in the magnon vacuum. Here, we omit the normal ordering of the quartic terms and assume that $\left\langle a_{i}^{\dagger} b_{j}\right\rangle=\left\langle a_{i} b_{j}^{\dagger}\right\rangle=\left\langle a_{i} a_{i}\right\rangle=$ $\left\langle a_{i}^{\dagger} a_{i}^{\dagger}\right\rangle=\left\langle b_{j}^{\dagger} b_{j}^{\dagger}\right\rangle=\left\langle b_{j} b_{j}\right\rangle=0$ due to the conservation of magnetization. After decomposing the quartic terms, 
we obtain a quadratic form spin-wave Hamiltonian in real space. Carrying out the Bogoliubov transformation, we can diagonalize the quadratic spin-wave Hamiltonian into

$$
H_{\mathrm{SW}}^{\prime}=\sum_{k=1}^{n_{\alpha}} \varepsilon_{k}^{(\alpha)} \alpha_{k}^{\dagger} \alpha_{k}+\sum_{l=1}^{n_{\beta}} \varepsilon_{l}^{(\beta)} \beta_{l}^{\dagger} \beta_{l}+E_{\mathrm{GS}}
$$

where $\varepsilon_{k}^{(\alpha)}\left[\varepsilon_{l}^{(\beta)}\right]$ is the eigenvalue of the bosonic mode $\alpha_{k}$ $\left(\beta_{l}\right), n_{\alpha}\left(n_{\beta}\right)$ is the number of the $\alpha_{k}\left(\beta_{l}\right)$ modes, and $E_{\mathrm{GS}}$ is the ground-state energy. Figure 2 shows the eigenvalues $\varepsilon_{k}^{(\alpha)}$ and $\varepsilon_{l}^{(\beta)}$ of Equation (6) in ascending order for three sizes of Penrose lattices. The eigenvalues become denser as the lattice size increases, and two gaps open near $\varepsilon \approx 2.2$ and $\varepsilon \approx 3.2$.

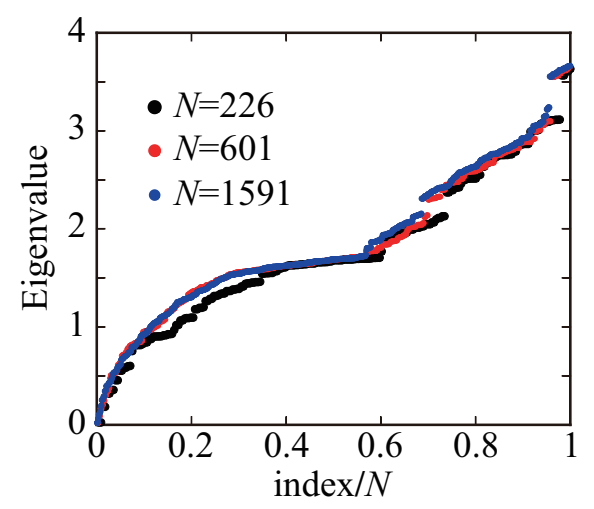

Figure 2 Eigenvalues of the Heisenberg antiferromagnet on the Penrose lattice of three different sizes $(N=226,601,1591)$ within spin-wave approximation. The horizontal axis is normalized by the size of the lattice.

\section{Effective magnetic Raman operator}

The magnetic Raman scattering is described by interaction between spins and photons. In this section, we follow a microscopic description of the magnetic Raman scattering, which is first given by Shastry and Shraiman [17-20], and present an effective magnetic Raman operator on the Penrose lattice. First, we consider a strongly correlated singleband Hubbard model:

$$
H_{\mathrm{Hb}}=H_{U}+H_{t}=U \sum_{i} n_{i \uparrow} n_{i \downarrow}-\sum_{i, j, \sigma} t_{i j} c_{i \sigma}^{\dagger} c_{j \sigma}
$$

where $c_{i \sigma}^{\dagger}\left(c_{i \sigma}\right)$ is the electron creation (annihilation) operator on site $i$ with spin $\sigma=\uparrow, \downarrow$ and $n_{i \sigma} \equiv c_{i \sigma}^{\dagger} c_{i \sigma} \cdot t_{i j}$ is the transfer integral, and $U(>0)$ is the on-site Coulomb repulsion. Hereafter, electron hopping is restricted to occur only between the nearest-neighbor sites.

The electron-photon coupling can be introduced by the Peierls substitution: $c_{i \sigma}^{\dagger} c_{j \sigma} \rightarrow c_{i \sigma}^{\dagger} c_{j \sigma} \exp \left(\frac{i e}{\hbar c} \int_{j}^{i} \boldsymbol{A} \cdot d \boldsymbol{r}\right)$, where $\boldsymbol{A}$ is the vector potential of the photon. We assume that incoming and outgoing photon wavelengths are much larger than lattice spacing. Then second-quantized vector potential is written as $\boldsymbol{A}=g_{\mathrm{in}} \boldsymbol{e}_{\mathrm{in}} \gamma_{\boldsymbol{k}_{\mathrm{in}}}+g_{\mathrm{sc}} \boldsymbol{e}_{\mathrm{sc}}^{*} \gamma_{\boldsymbol{k}_{\mathrm{sc}}}^{\dagger}$ where $g_{\text {in }}=\sqrt{h c^{2} / \omega_{\text {in }} V}$ and $g_{\mathrm{sc}}=\sqrt{h c^{2} / \omega_{\mathrm{sc}} V}$ with volume $V . \omega_{\text {in }}\left(\omega_{\mathrm{sc}}\right), \boldsymbol{k}_{\mathrm{in}}\left(\boldsymbol{k}_{\mathrm{sc}}\right)$, and $\boldsymbol{e}_{\mathrm{in}}\left(\boldsymbol{e}_{\mathrm{sc}}\right)$ stand for frequency, momentum, and polarization of incident (scattered) photon, respectively. $\gamma^{\dagger}(\gamma)$ denotes the photon creation (annihilation) operator. Expanding the exponential of the hopping terms and extracting the first-order term of the vector potential, the current operator reads

$$
H_{\mathrm{c}}=-\frac{i e}{\hbar c} \sum_{i, j, \sigma} t_{i j} \boldsymbol{A} \cdot \boldsymbol{\delta}_{i j} c_{i \sigma}^{\dagger} c_{j \sigma}
$$

where $\boldsymbol{\delta}_{i j}$ is the vector connecting sites $i$ and $j$.

Since the Raman process is made of two photons (one photon in, one photon out), only the second-order process of $\boldsymbol{A}$ is required. We are interested in a half-filled $\left(\sum_{\sigma}\left\langle n_{i \sigma}\right\rangle=1\right)$ and localized $(U \gg t)$ system. In this situation, both the initial and final states belong to the groundstate manifold of singly occupied states, and $H_{\mathrm{c}}$ and $H_{t}$ can be treated as a perturbation. The initial states are taken to be a direct product of singly occupied electron states with incident photon. When $H_{c}$ is operated there, the intermediate states are described as electron states with one holon and one doublon, and photon states with zero or two photons. The energy gap between the initial and intermediate states is $U-\hbar \omega_{\text {in }}$ for no photons and $U+\hbar \omega_{\mathrm{sc}}$ for two photons, respectively. Considering a situation where the photon energy is non-negligible with respect to $U$ and assuming $U+\hbar \omega_{\mathrm{sc}} \gg U \gg U-\hbar \omega_{\text {in }}>t$, the intermediate states with no photons are dominant. Under this condition, the effective Raman operator reads

$$
\begin{aligned}
\mathcal{R} & =\mathcal{P} H_{\mathrm{c}} \frac{1}{\varepsilon_{i}-H_{U}-H_{t}} H_{\mathrm{c}} \mathcal{P} \\
& =\mathcal{P} H_{\mathrm{c}} \frac{1}{\varepsilon_{i}-H_{U}} \sum_{n=0}^{\infty}\left(H_{t} \frac{1}{\varepsilon_{i}-H_{U}}\right)^{n} H_{\mathrm{c}} \mathcal{P}
\end{aligned}
$$

where $\varepsilon_{i}$ is the initial-state energy and $\mathcal{P}$ is a projection operator to the spin- $1 / 2$ sector. Because of the electronhole symmetry in the half-filled band, any term of odd $n$ vanishes in Equation (9). Furthermore, $\left(\varepsilon_{i}-H_{U}\right)^{-1}=$ $\left(\hbar \omega_{\text {in }}-U\right)^{-1}$ becomes a $c$-number. Finally, we convert the electron operators into $S=1 / 2$ spin operators using the following projection:

$$
\mathcal{P} c_{i \sigma}^{\dagger} c_{i \sigma^{\prime}} \mathcal{P}=\frac{1}{2} \delta_{\sigma^{\prime}, \sigma}+\boldsymbol{S}_{i} \cdot \boldsymbol{\tau}_{\sigma^{\prime} \sigma}
$$

where $\tau$ is the Pauli matrix.

The second-order perturbation is the lowest nonvanishing order in the Shastry-Shraiman formulation. It corresponds to the $n=0$ term in Equation (9) and gives the Loudon-Fleury magnetic Raman operator [10]:

$$
\mathcal{R}^{(2)}=\sum_{\langle i, j\rangle} \frac{4 t^{2}}{U-\hbar \omega_{\mathrm{in}}}\left(\boldsymbol{e}_{\mathrm{in}} \cdot \boldsymbol{\delta}_{i j}\right)\left(\boldsymbol{e}_{\mathrm{sc}}^{*} \cdot \boldsymbol{\delta}_{i j}\right) \boldsymbol{S}_{i} \cdot \boldsymbol{S}_{j}
$$


Here, we omit some constants that do not affect the Raman intensity. The second-order Raman operator is dominant in the case of $U-\hbar \omega_{\text {in }} \gg t$.

The fourth-order effective Raman operator is the next nonvanishing order. It corresponds to $n=2$ in Equation (9). The fourth-order magnetic Raman operator includes the scalar-spin-chirality terms $\boldsymbol{S}_{i} \cdot\left(\boldsymbol{S}_{j} \times \boldsymbol{S}_{k}\right)$ and/or the ring-exchange terms $\left(\boldsymbol{S}_{i} \cdot \boldsymbol{S}_{j}\right)\left(\boldsymbol{S}_{k} \cdot \boldsymbol{S}_{l}\right)$. The prefactor of the fourth-order magnetic Raman operator is $t^{4} /\left(U-\hbar \omega_{\text {in }}\right)^{3}$. For details about the fourth-order magnetic Raman operator, see Appendix A. If the incident photon energy $\hbar \omega_{\text {in }}$ approaches to the resonant region $U-\hbar \omega_{\text {in }} \sim O(t)$, fourthorder contributions can manifest in the Raman intensity.

For theoretical calculations, it is convenient to decompose the polarization dependence of the magnetic Raman spectrum into the irreducible representations (irreps) of the lattice point group. The point group of the Penrose lattice is $\mathbf{C}_{5 \mathrm{v}}$. In $\mathbf{C}_{5 \mathrm{v}}$, the polarization dependence of the Ramanactive modes decomposes into two one-dimensional irreps $A_{1}$ and $A_{2}$, and one two-dimensional irrep $E_{2}$ as follows:

$$
\begin{aligned}
A_{1} & : e_{\mathrm{in}}^{x} e_{\mathrm{sc}}^{* x}+e_{\mathrm{in}}^{y} e_{\mathrm{sc}}^{* y} \\
A_{2} & : e_{\mathrm{in}}^{x} e_{\mathrm{sc}}^{* y}-e_{\mathrm{in}}^{y} e_{\mathrm{sc}}^{* x} \\
E_{2}^{(1)} & : e_{\mathrm{in}}^{x} e_{\mathrm{sc}}^{* x}-e_{\mathrm{in}}^{y} e_{\mathrm{sc}}^{* y} \\
E_{2}^{(2)} & : e_{\mathrm{in}}^{x} e_{\mathrm{sc}}^{* y}+e_{\mathrm{in}}^{y} e_{\mathrm{sc}}^{* x}
\end{aligned}
$$

The magnetic Raman spectrum at $T=0$ is given by Fermi's golden rule:

$$
I(\omega)=\sum_{n}\left|\left\langle\Psi_{n}|\mathcal{R}| \Psi_{0}\right\rangle\right|^{2} \delta\left(\hbar \omega-E_{n}+E_{0}\right)
$$

where $\left|\Psi_{0}\right\rangle$ is a ground state of the Heisenberg model, $\left|\Psi_{n}\right\rangle$ is excited states. $E_{0}$ and $E_{n}$ are eigenvalues of the ground and excited states, respectively.

\section{Results \\ 4.1 Second-order magnetic Raman intensity: Within the Loudon-Fleury mechanism}

First, we consider the Raman spectrum within the Loudon-Fleury mechanism. In this section, we use the second-order magnetic Raman operator [Equation (11)]. We consider a bosonic representation of the spin operators in Equation (11) by using the Holstein-Primakoff transformation. In this study, we consider the two-magnon scattering. It corresponds to the expansion of the magnetic Raman operator up to the bosonic two-body terms.

Figure 3 shows the spin-wave result of the two-magnon scattering Raman intensity within the second-order Raman operator for the $N=601$ sites cluster of the Penrose lattice. We find that the second-order Raman intensity comes from the $E_{2}$ representation and shows no linear polarization dependence. To understand this depolarization, we set the incident and scattered polarization vectors as

$$
\boldsymbol{e}_{\mathrm{in}}=\left(\cos \theta_{\mathrm{in}}, \sin \theta_{\mathrm{in}}\right), \quad \boldsymbol{e}_{\mathrm{sc}}=\left(\cos \theta_{\mathrm{sc}}, \sin \theta_{\mathrm{sc}}\right)
$$

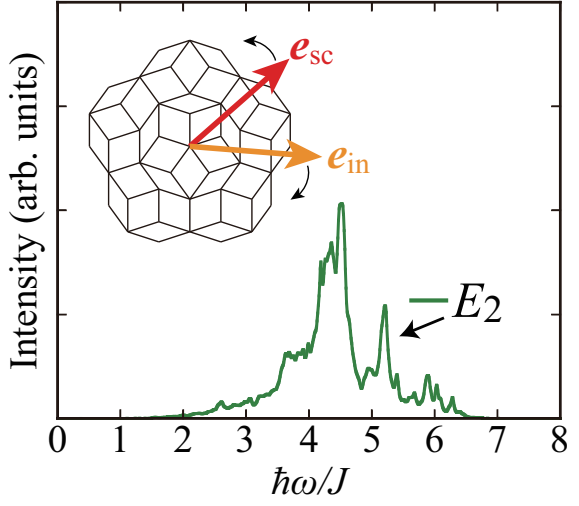

Figure 3 Two-magnon scattering magnetic Raman spectrum of the $N=601$ sites cluster Penrose lattice Heisenberg antiferromagnet within the second-order magnetic Raman operator. The spectrum arises from the $E_{2}$ representation of the $\mathbf{C}_{5 \mathrm{v}}$ point group, and does not depend on the incident and scattered photon polarizations $\boldsymbol{e}_{\mathrm{in}}$ and $\boldsymbol{e}_{\mathrm{sc}}$.

where $\theta_{\text {in }}$ and $\theta_{\mathrm{sc}}$ are the angles of the polarization vectors of the incident and scattered photons with respect to the $x$ axis. Under this condition, the $E_{2}$ mode Raman spectrum is written as

$$
\begin{aligned}
I\left(\omega, \theta_{\mathrm{in}}, \theta_{\mathrm{sc}}\right)= & \sum_{n} \mid\left\langle\Psi_{n}\right| \mathcal{R}_{E_{2}^{(1)}} \cos \left(\theta_{\mathrm{in}}+\theta_{\mathrm{sc}}\right) \\
& +\left.\mathcal{R}_{E_{2}^{(2)}} \sin \left(\theta_{\mathrm{in}}+\theta_{\mathrm{sc}}\right)\left|\Psi_{0}\right\rangle\right|^{2} \\
& \times \delta\left(\hbar \omega-E_{n}+E_{0}\right)
\end{aligned}
$$

where $\mathcal{R}_{E_{2}^{(1)}}$ and $\mathcal{R}_{E_{2}^{(2)}}$ are magnetic Raman operators obtained by irreducible decomposition for the first and second components of the $E_{2}$ representation, respectively. $\mathcal{R}_{E_{2}^{(1)}}$ and $\mathcal{R}_{E_{2}^{(2)}}$ are orthogonal to each other. Scattering intensities of $\mathcal{R}_{E_{2}^{(1)}}$ and $\mathcal{R}_{E_{2}^{(2)}}$ are degenerate. Therefore, the $E_{2}$ mode Raman spectrum is independent of polarization angles:

$$
\begin{aligned}
I\left(\omega, \theta_{\text {in }}, \theta_{\mathrm{sc}}\right)= & \cos ^{2}\left(\theta_{\mathrm{in}}+\theta_{\mathrm{sc}}\right) I_{E_{2}}(\omega) \\
& +\sin ^{2}\left(\theta_{\mathrm{in}}+\theta_{\mathrm{sc}}\right) I_{E_{2}}(\omega)=I_{E_{2}}(\omega)
\end{aligned}
$$

where $I_{E_{2}}(\omega)$ denotes intensity of the $E_{2}$ mode.

4.2 Fourth-order magnetic Raman intensity: Beyond the Loudon-Fleury mechanism

Next, we calculate the two-magnon scattering Raman intensity of the fourth-order magnetic Raman operator. We consider two polarizations, one is called $x x$ polarization corresponding to $\left(\theta_{\mathrm{in}}, \theta_{\mathrm{sc}}\right)=(0,0)$, and the other is called $x y$ polarization that corresponds to $\left(\theta_{\mathrm{in}}, \theta_{\mathrm{sc}}\right)=(0, \pi / 2)$. As shown in Figure 4, the fourth-order Raman operators yield spectral weight of the $A_{1}$ mode in the $x x$ polarization [Figure 4(a)], and the $A_{2}$ mode in the $x y$ polarization [Figure 4(b)], as well as the linearly polarization indepen- 

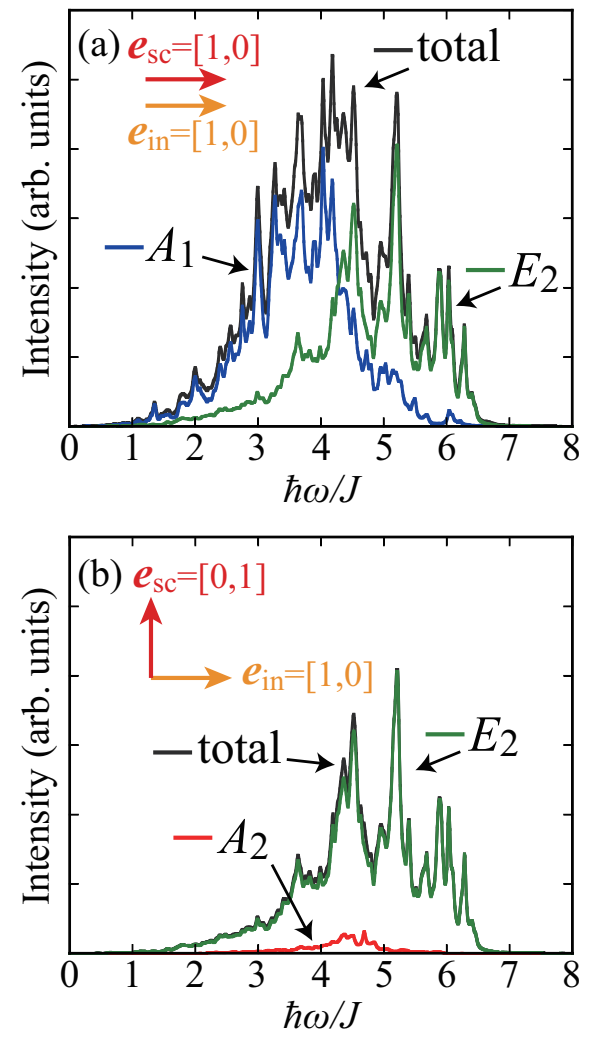

Figure 4 Two-magnon scattering magnetic Raman spectra of the fourth-order magnetic Raman operator on the $N=601$ sites cluster for $x x$ polarization (a) and $x y$ polarization (b).

dent $E_{2}$ mode. The observed spectrum for each polarization is described as $I_{x x}(\omega)=I_{A_{1}}(\omega)+I_{E_{2}}(\omega)$ for the $x x$ polarization, and $I_{x y}(\omega)=I_{A_{2}}(\omega)+I_{E_{2}}(\omega)$ for the $x y$ polarization. In general, the linear polarization dependence of the fourth-order Raman intensity is given by

$$
\begin{aligned}
I\left(\omega, \theta_{\text {in }}, \theta_{\mathrm{sc}}\right)= & \cos ^{2}\left(\theta_{\text {in }}-\theta_{\mathrm{sc}}\right) I_{A_{1}}(\omega) \\
& +\sin ^{2}\left(\theta_{\text {in }}-\theta_{\mathrm{sc}}\right) I_{A_{2}}(\omega)+I_{E_{2}}(\omega)
\end{aligned}
$$

As shown in Equation (17), the fourth-order Raman spectrum is observed as a superposition of Raman-active modes. We employ two linearly and one circularly polarized lights to decompose the Raman intensities into all the symmetry species from observations. In order to separate all the irreducible components of the spectrum, only linearly polarized light is insufficient, and circularly polarized light is required. The Raman intensities of each irreducible representation are obtained as follows:

$$
\begin{aligned}
& I_{A_{1}}(\omega)=I_{x x}(\omega)-\frac{1}{2} I_{L R}(\omega) \\
& I_{A_{2}}(\omega)=I_{x y}(\omega)-\frac{1}{2} I_{L R}(\omega) \\
& I_{E_{2}}(\omega)=\frac{1}{2} I_{L R}(\omega)
\end{aligned}
$$

where $I_{L R}(\omega)$ is the $L R$ polarization Raman intensity. The $L R$ polarization refers to left circularly polarized incident photon $\boldsymbol{e}_{\mathrm{in}}=\frac{1}{\sqrt{2}}(1, i)$ and right circularly polarized scattered photon $\boldsymbol{e}_{\mathrm{sc}}=\frac{1}{\sqrt{2}}(1,-i)$.

The fourth-order magnetic Raman spectra include several magnetic excitation contributions. In particular, the $A_{2}$ mode spectrum provides a direct observation of dynamic spin-chirality fluctuations. The geometry of the lattice plays an important role in the observation of spinchirality fluctuations through Raman scattering. The spinchirality terms of the fourth-order magnetic Raman operator cancel out on the two types of fourth-order electron hopping pathways: (1) four-site loop pathway and (2) three-site straight pathway. As an example, we consider a two-dimensional periodic lattice with a single-site unit cell. This lattice has only two independent primitive lattice vectors. It always satisfies the conditions to cancel the spin-chirality terms. However, this is not the case with the Penrose lattice. Because of the quasiperiodicity, the Penrose lattice has four independent primitive lattice vectors with greater than the lattice dimension $d=2$. This allows extra fourth-order electron hopping pathways. Therefore, the spin-chirality-driven $A_{2}$ mode spectrum can survive in the Penrose lattice.

\subsection{Effects of magnon-magnon interactions}

To compare the spin-wave results with the Lanczös exact diagonalization results, we consider a small-sized $(N=$ 16 ), fivefold rotationally symmetric Penrose lattice cluster. In the Lanczös method, the Raman spectrum is obtained from a continued fraction:

$$
I(\omega)=-\frac{1}{\pi} \operatorname{Im}\left\{\left\langle\Psi_{0}\left|\mathcal{R}^{\dagger} \frac{1}{\hbar \omega+E_{0}+i \eta-H} \mathcal{R}\right| \Psi_{0}\right\rangle\right\}
$$

where $\eta$ is a small imaginary part added to give a finite damping of the $\delta$-functions.

In the spin-wave calculation, we incorporate the magnon-magnon interactions by the configuration interaction (CI) method. We apply the two-magnon excitation CI method in this study. We consider a zero-magnon state $|0 \mathrm{M}\rangle$ and two-magnon excited states $|2 \mathrm{M}\rangle$ [21]:

$$
|0 \mathrm{M}\rangle=|0\rangle, \quad|2 \mathrm{M}(k, l)\rangle=\alpha_{k}^{\dagger} \beta_{l}^{\dagger}|0\rangle
$$

where $|0\rangle$ denotes the magnon-vacuum state. Spin-wave eigenstates are improved as

$$
\left|\Psi_{n}\right\rangle_{\mathrm{CI}}=c_{0, n}|0\rangle+\sum_{k, l} c_{(k, l), n} \alpha_{k}^{\dagger} \beta_{l}^{\dagger}|0\rangle
$$

where coefficients $c_{0, n}$ and $c_{(k, l), n}$ are components of the eigenvector obtained by diagonalizing the two-magnon excitation CI Hamiltonian matrix:

$$
H_{\mathrm{CI}}=\left[\begin{array}{ll}
\left\langle 0 \mathrm{M}\left|H_{\mathrm{SW}}\right| 0 \mathrm{M}\right\rangle & \left\langle 0 \mathrm{M}\left|H_{\mathrm{SW}}\right| 2 \mathrm{M}\right\rangle \\
\left\langle 2 \mathrm{M}\left|H_{\mathrm{SW}}\right| 0 \mathrm{M}\right\rangle & \left\langle 2 \mathrm{M}\left|H_{\mathrm{SW}}\right| 2 \mathrm{M}\right\rangle
\end{array}\right]
$$



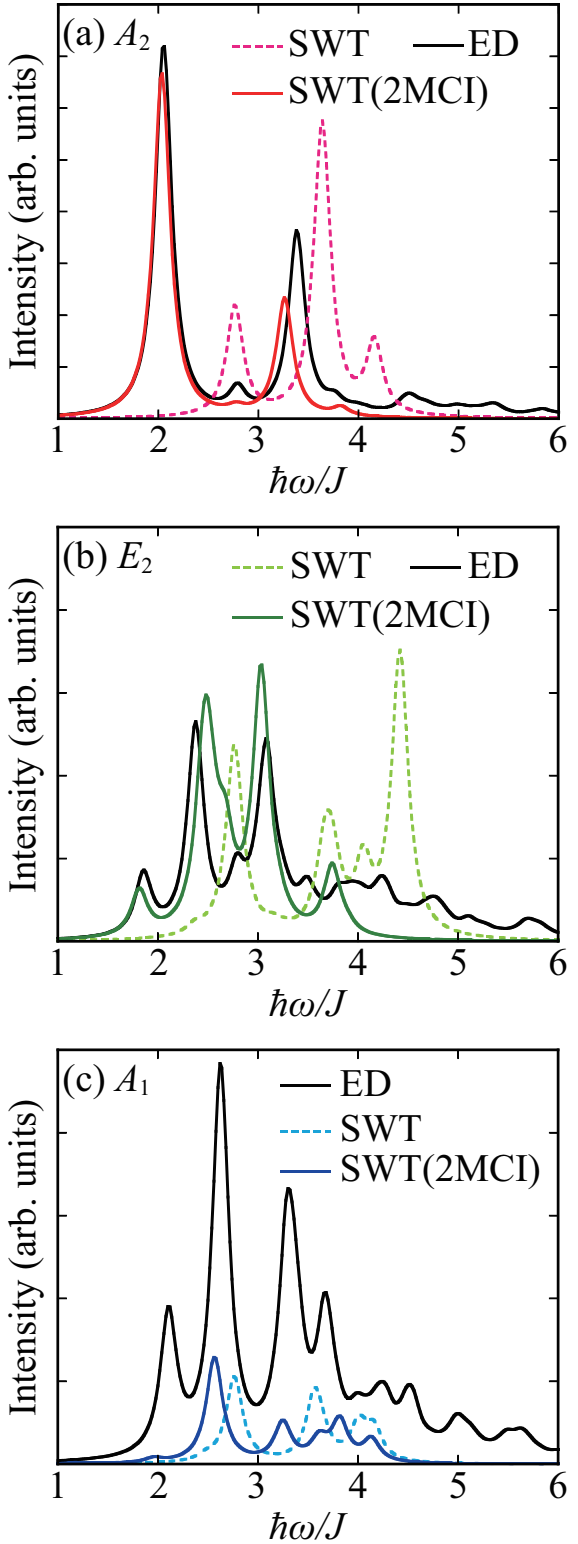

Figure 5 The fourth-order magnetic Raman spectra on the $N=$ 16 sites cluster for (a) $A_{2}$ mode, (b) $E_{2}$ mode, and (c) $A_{1}$ mode. Spectra are calculated by Lanczös exact diagonalization (ED), spin-wave theory without magnon-magnon interactions (SWT), and spin-wave theory with magnon-magnon interactions introduced by two-magnon excitation CI method [SWT(2MCI)].

We note that the two-magnon excitation CI calculation corresponds to solving the ladder-approximation BetheSalpeter equation with quartic magnon interaction terms [12].

Results are shown in Figure 5. First, we focus on the $A_{2}$ mode spectra [Figure 5(a)]. Comparing the spin-wave results in the absence and presence of the magnon-magnon interactions, we find that the interaction shifts the Raman intensity peak to the lower energy side. The line shapes and peak positions of the interacting spin-wave result are in good agreement with the exact diagonalization result. We conclude that the spin-wave calculation of the two-magnon scattering process can describe the spin-chirality-driven $A_{2}$ mode magnetic Raman spectrum very well.

Figure 5(b) shows the $E_{2}$ mode spectra. We find that the two-magnon scattering intensity of the spin-wave theory with the magnon-magnon interactions agrees well with the exact diagonalization result in the low-frequency (about $\hbar \omega<4 J$ ) region compared to the case without the interaction. However, even the interacting spin-wave result lacks the high-frequency tail of the exact result. This is expected to be explained by contributions from higher-order magnon scattering.

On the other hand, as shown in Figure 5(c), the $A_{1}$ mode magnetic Raman spectra of the exact diagonalization and the spin-waves are not consistent. The two-magnon scattering spin-wave spectra are considerably smaller than the exact spectrum, even when the magnon-magnon interactions are included. This suggests that the higher order multimagnon scattering, for instance four-magnon scattering, is dominant in the $A_{1}$ mode Raman intensity.

\section{Conclusion}

We have presented the magnetic Raman spectra of the two-dimensional $\mathbf{C}_{5 \mathrm{v}}$ Penrose lattice Heisenberg antiferromagnet. The Raman intensity within the Loudon-Fleury mechanism arises from the $E_{2}$ mode and shows no linear polarization dependence due to the degeneracy of the twodimensional irreducible representation $E_{2}$. In contrast, the fourth-order Raman intensities consist of $A_{1}$ and $A_{2}$, as well as $E_{2}$, modes and therefore yield strong polarization dependence. The $A_{2}$ mode spectrum is driven by scalarspin-chirality terms. It is arisen from quasiperiodic structure of the Penrose lattice. We can separately extract all the symmetry species from observations with the use of two linearly and one circularly polarized lights. The twomagnon scattering with the magnon-magnon interactions can describe the $A_{2}$ and $E_{2}$ mode spectra very well. This means that the spin-chirality excitations and exchange excitations can be understood by the two-magnon scattering process. In contrast, the $A_{1}$ mode spectrum, which is mainly due to the ring-exchange excitations, are not consistent with the two-magnon scattering results, even when the magnon-magnon interactions are incorporated. Considering multimagnon scattering processes and higher-order interactions is left for future investigation.

Acknowledgements We thank J. Ohara and Y. Noriki for fruitful discussion. This study is supported by the Ministry of Education, Culture, Sports, Science, and Technology of Japan.

Appendix A: The fourth-order effective magnetic Raman operator

The fourth-order effective magnetic Raman operator is 
written as

$$
\begin{aligned}
& \mathcal{R}^{(4)}=\sum_{\langle 1,2,3,4\rangle} \frac{t^{4}}{\left(U-\hbar \omega_{\text {in }}\right)^{3}}\{ \\
& -4 \sum_{n=1}^{4}\left(\boldsymbol{e}_{\mathrm{in}} \cdot \boldsymbol{\delta}_{n}\right)\left(\boldsymbol{e}_{\mathrm{sc}}^{*} \cdot\left[\boldsymbol{\delta}_{n+1}+2 \boldsymbol{\delta}_{n+2}+\boldsymbol{\delta}_{n+3}\right]\right) \\
& \times\left[\mathcal{Q}_{1234}+\mathcal{Q}_{1432}-\mathcal{Q}_{1324}\right] \\
& +2 i \sum_{n=1}^{4} \Delta_{n}^{\mathrm{ch}} \boldsymbol{S}_{n+2} \cdot\left(\boldsymbol{S}_{n+1} \times \boldsymbol{S}_{n}\right) \\
& \left.+\sum_{n=1}^{4} \Delta_{n}^{\mathrm{ex}} \boldsymbol{S}_{n} \cdot \boldsymbol{S}_{n+1}+\sum_{n=1}^{2} \Delta_{n}^{\mathrm{ex}^{\prime}} \boldsymbol{S}_{n} \cdot \boldsymbol{S}_{n+2}\right\} \\
& +\sum_{\langle 1,2,3\rangle} \frac{t^{4}}{\left(U-\hbar \omega_{\text {in }}\right)^{3}}\{ \\
& 4 i\left[\left(\boldsymbol{e}_{\mathrm{in}} \cdot \boldsymbol{\delta}_{1}\right)\left(\boldsymbol{e}_{\mathrm{sc}}^{*} \cdot \boldsymbol{\delta}_{2}\right)-\left(\boldsymbol{e}_{\mathrm{in}} \cdot \boldsymbol{\delta}_{2}\right)\left(\boldsymbol{e}_{\mathrm{sc}}^{*} \cdot \boldsymbol{\delta}_{1}\right)\right] \\
& \times \boldsymbol{S}_{3} \cdot\left(\boldsymbol{S}_{2} \times \boldsymbol{S}_{1}\right) \\
& +2 \sum_{n=1}^{2} \tilde{\Delta}_{n}^{\mathrm{ex}} \boldsymbol{S}_{n} \cdot \boldsymbol{S}_{n+1} \\
& -2\left[\left(\boldsymbol{e}_{\mathrm{in}} \cdot \boldsymbol{\delta}_{1}\right)\left(\boldsymbol{e}_{\mathrm{sc}}^{*} \cdot \boldsymbol{\delta}_{2}\right)+\left(\boldsymbol{e}_{\mathrm{in}} \cdot \boldsymbol{\delta}_{2}\right)\left(\boldsymbol{e}_{\mathrm{sc}}^{*} \cdot \boldsymbol{\delta}_{1}\right)\right] \\
& \left.\times \boldsymbol{S}_{1} \cdot \boldsymbol{S}_{3}\right\} \\
& \mathcal{Q}_{i j k l} \equiv\left(\boldsymbol{S}_{i} \cdot \boldsymbol{S}_{j}\right)\left(\boldsymbol{S}_{k} \cdot \boldsymbol{S}_{l}\right) \\
& \begin{aligned}
\Delta_{n}^{\mathrm{ch}} \equiv & \left(\boldsymbol{e}_{\mathrm{in}} \cdot \boldsymbol{\delta}_{n}\right)\left(\boldsymbol{e}_{\mathrm{sc}}^{*} \cdot\left[-\boldsymbol{\delta}_{n+1}-2 \boldsymbol{\delta}_{n+2}+\boldsymbol{\delta}_{n+3}\right]\right) \\
& +\left(\boldsymbol{e}_{\mathrm{in}} \cdot \boldsymbol{\delta}_{n+1}\right)\left(\boldsymbol{e}_{\mathrm{sc}}^{*} \cdot\left[-\boldsymbol{\delta}_{n+2}+2 \boldsymbol{\delta}_{n+3}+\boldsymbol{\delta}_{n}\right]\right) \\
& +\left(\boldsymbol{e}_{\mathrm{in}} \cdot \boldsymbol{\delta}_{n+2}\right)\left(\boldsymbol{e}_{\mathrm{sc}}^{*} \cdot\left[\boldsymbol{\delta}_{n+3}+2 \boldsymbol{\delta}_{n}+\boldsymbol{\delta}_{n+1}\right]\right) \\
& +\left(\boldsymbol{e}_{\mathrm{in}} \cdot \boldsymbol{\delta}_{n+3}\right)\left(\boldsymbol{e}_{\mathrm{sc}}^{*} \cdot\left[-\boldsymbol{\delta}_{n}-2 \boldsymbol{\delta}_{n+1}-\boldsymbol{\delta}_{n+2}\right]\right)
\end{aligned} \\
& \Delta_{n}^{\mathrm{ex}} \equiv\left(\boldsymbol{e}_{\mathrm{in}} \cdot \boldsymbol{\delta}_{n}\right)\left(\boldsymbol{e}_{\mathrm{sc}}^{*} \cdot\left[-\boldsymbol{\delta}_{n+1}+2 \boldsymbol{\delta}_{n+2}-\boldsymbol{\delta}_{n+3}\right]\right) \\
& +\left(\boldsymbol{e}_{\mathrm{in}} \cdot \boldsymbol{\delta}_{n+1}\right)\left(\boldsymbol{e}_{\mathrm{sc}}^{*} \cdot\left[\boldsymbol{\delta}_{n+2}-2 \boldsymbol{\delta}_{n+3}-\boldsymbol{\delta}_{n}\right]\right) \\
& +\left(\boldsymbol{e}_{\mathrm{in}} \cdot \boldsymbol{\delta}_{n+2}\right)\left(\boldsymbol{e}_{\mathrm{sc}}^{*} \cdot\left[\boldsymbol{\delta}_{n+3}+2 \boldsymbol{\delta}_{n}+\boldsymbol{\delta}_{n+1}\right]\right) \\
& +\left(\boldsymbol{e}_{\mathrm{in}} \cdot \boldsymbol{\delta}_{n+3}\right)\left(\boldsymbol{e}_{\mathrm{sc}}^{*} \cdot\left[-\boldsymbol{\delta}_{n}-2 \boldsymbol{\delta}_{n+1}+\boldsymbol{\delta}_{n+2}\right]\right) \\
& \Delta_{n}^{\mathrm{ex}^{\prime}} \equiv\left(\boldsymbol{e}_{\mathrm{in}} \cdot \boldsymbol{\delta}_{n}\right)\left(\boldsymbol{e}_{\mathrm{sc}}^{*} \cdot\left[\boldsymbol{\delta}_{n+1}+2 \boldsymbol{\delta}_{n+2}-\boldsymbol{\delta}_{n+3}\right]\right) \\
& +\left(\boldsymbol{e}_{\mathrm{in}} \cdot \boldsymbol{\delta}_{n+1}\right)\left(\boldsymbol{e}_{\mathrm{sc}}^{*} \cdot\left[-\boldsymbol{\delta}_{n+2}+2 \boldsymbol{\delta}_{n+3}+\boldsymbol{\delta}_{n}\right]\right) \\
& +\left(\boldsymbol{e}_{\mathrm{in}} \cdot \boldsymbol{\delta}_{n+2}\right)\left(\boldsymbol{e}_{\mathrm{sc}}^{*} \cdot\left[\boldsymbol{\delta}_{n+3}+2 \boldsymbol{\delta}_{n}-\boldsymbol{\delta}_{n+1}\right]\right) \\
& +\left(\boldsymbol{e}_{\mathrm{in}} \cdot \boldsymbol{\delta}_{n+3}\right)\left(\boldsymbol{e}_{\mathrm{sc}} \cdot\left[-\boldsymbol{\delta}_{n}+2 \boldsymbol{\delta}_{n+1}+\boldsymbol{\delta}_{n+2}\right]\right) \\
& \begin{aligned}
\tilde{\Delta}_{n}^{\mathrm{ex}} \equiv & \left(\boldsymbol{e}_{\mathrm{in}} \cdot \boldsymbol{\delta}_{n}\right)\left(\boldsymbol{e}_{\mathrm{sc}}^{*} \cdot\left[\boldsymbol{\delta}_{1}+\boldsymbol{\delta}_{2}\right]\right) \\
& +\left(\boldsymbol{e}_{\mathrm{in}} \cdot\left[\boldsymbol{\delta}_{1}+\boldsymbol{\delta}_{2}\right]\right)\left(\boldsymbol{e}_{\mathrm{sc}}^{*} \cdot \boldsymbol{\delta}_{n}\right)
\end{aligned}
\end{aligned}
$$

where $\sum_{\langle 1,2,3,4\rangle}$ is taken over four-sites loop pathways, and $\sum_{\langle 1,2,3\rangle}$ is taken over three-sites linked pathways (Figure 6). $e_{\mathrm{in}}$ and $e_{\mathrm{sc}}^{*}$ are the polarization vectors of incident and scattered photons. $\boldsymbol{\delta}_{n} \equiv \boldsymbol{r}_{n+1}-\boldsymbol{r}_{n}$ is the vector connecting sites $n$ and $n+1$. We set $n \equiv n+4(\bmod 4)$.

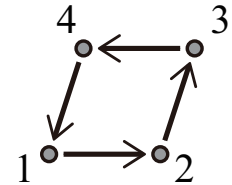

(a)

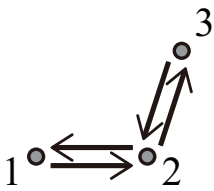

\begin{tabular}{|c|c|c|}
\hline & $C_{5}^{n}$ & $\sigma_{n}$ \\
\hline$A_{1}$ & 1 & 1 \\
\hline$A_{2}$ & 1 & -1 \\
\hline$E_{1}$ & $\left(\begin{array}{cc}\cos \theta_{n} & \sin \theta_{n} \\
-\sin \theta_{n} & \cos \theta_{n}\end{array}\right)$ & $\left(\begin{array}{cc}\cos 2 \alpha_{n} & \sin 2 \alpha_{n} \\
\sin 2 \alpha_{n} & -\cos 2 \alpha_{n}\end{array}\right)$ \\
\hline$E_{2}$ & $\left(\begin{array}{cc}\cos 2 \theta_{n} & \sin 2 \theta_{n} \\
\sin 2 \theta_{n} & \cos 2 \theta_{n}\end{array}\right)$ & $\left(\begin{array}{cc}\cos 4 \alpha_{n} & \sin 4 \alpha_{n} \\
\sin 4 \alpha_{n} & -\cos 4 \alpha_{n}\end{array}\right)$ \\
\hline \multicolumn{3}{|c|}{$\theta_{n}=\frac{2 \pi}{5} n, \alpha_{n}=\frac{2 \pi}{5} n-\frac{\pi}{10}, n=1,2,3,4,5$} \\
\hline
\end{tabular}

(b)
Figure 6 Two types of fourth-order electron hopping pathways. (a) Four-site loop pathway and (b) three-site pathway. Arrows denote the movement of electrons arising from $H_{c}$ and $H_{t}$.

\section{Appendix B: Irreducible decomposition of the} magnetic Raman operator

The projection operator for the irreducible representation (irrep) $\beta$ of group $\mathcal{G}$ is given by

$$
P_{l(m)}^{(\beta)}=\frac{d_{\beta}}{g} \sum_{R} D_{l m}^{(\beta)}(R)^{*} R
$$

where $d_{\beta}$ is a dimension of the irrep $\beta, g$ is an order of group $\mathcal{G}, R$ is a symmetry operation, and $D_{l m}^{(\beta)}(R)$ is a unitary representation matrix of $R$, respectively. Table 1 shows the representation matrix of the $\mathbf{C}_{5 \mathrm{v}}$ point group. In this case, the $A_{1}, A_{2}$, and $E_{2}$ representations can be Raman active [22]. The magnetic Raman operator decomposes into two one-dimensional irreps $A_{1}$ and $A_{2}$, and one two-dimensional irrep $E_{2}$,

$$
\begin{aligned}
\mathcal{R}_{A_{1}} & =P^{\left(A_{1}\right)} \mathcal{R}=E_{A_{1}} O_{A_{1}} \\
\mathcal{R}_{A_{2}} & =P^{\left(A_{2}\right)} \mathcal{R}=E_{A_{2}} O_{A_{2}} \\
\mathcal{R}_{E_{2}^{(1)}} & =P_{1(1)}^{\left(E_{2}\right)} \mathcal{R}=E_{E_{2}^{(1)}} O_{E_{2}^{(1)}} \\
\mathcal{R}_{E_{2}^{(2)}} & =P_{2(2)}^{\left(E_{2}\right)} \mathcal{R}=E_{E_{2}^{(2)}} O_{E_{2}^{(2)}}
\end{aligned}
$$

where $E_{A_{1}}=e_{\mathrm{in}}^{x} e_{\mathrm{sc}}^{* x}+e_{\mathrm{in}}^{y} e_{\mathrm{sc}}^{* y}, E_{A_{2}}=e_{\mathrm{in}}^{x} e_{\mathrm{sc}}^{* y}-e_{\mathrm{in}}^{y} e_{\mathrm{sc}}^{* x}$, $E_{E_{2}^{(1)}}=e_{\mathrm{in}}^{x} e_{\mathrm{sc}}^{* x}-e_{\mathrm{in}}^{y} e_{\mathrm{sc}}^{* y}$, and $E_{E_{2}^{(2)}}=e_{\mathrm{in}}^{x} e_{\mathrm{sc}}^{* y}+e_{\mathrm{in}}^{y} e_{\mathrm{sc}}^{* x}$

Table 1 The representation matrix of the $\mathbf{C}_{5 \mathrm{v}}$ point group. 
are polarization dependence, and $O_{A_{1}}, O_{A_{2}}, O_{E_{2}^{(1)}}$, and $O_{E_{2}^{(2)}}$ are linear combinations of spin operators in each irreducible representation.

In the second-order magnetic Raman operator, the spin operator part is represented by the nearest-neighbor exchange coupling, as shown in Equation (11). Since the $A_{1}$ representation matrix is unity for all symmetry operations, $O_{A_{1}}$ commutes with the Hamiltonian. Therefore, the $A_{1}$ mode spectrum does not appear. Next, if we consider the time reversal operation of Raman processes, this is equivalent to swapping the creation and annihilation of photons and reversing the hopping path of electrons. As a result, $\boldsymbol{e}_{\mathrm{in}}$ and $\boldsymbol{e}_{\mathrm{sc}}^{*}$ are replaced in the polarization dependence and site indices are reversed in the spin operators. The spin operator parts are even under this operation for all irreducible representations. However, $E_{A_{2}}$ is odd under this operation (other modes are even), $A_{2}$ mode is unsuitable because it is not even in its entirety. For the above reasons, there is only $E_{2}$ mode in the second-order Raman intensity.

On the other hand, in the fourth-order magnetic Raman operator, $A_{1}$ and $A_{2}$ modes can become Raman active as well as $E_{2}$ mode. This is due to next-nearest-neighbor exchange, ring-exchange, and spin-chirality terms.

\section{References}

[1] D. Schehtman, I. Blech, D. Gratias, and J. W. Cahn, Phys. Rev. Lett. 53, 1951 (1984).

[2] M. Kohmoto and B. Sutherland, Phys. Rev. Lett. 56, 2740 (1986).

[3] M. Arai, T. Tokihiro, T. Fujiwara, and M. Kohmoto, Phys. Rev. B 38, 1621.

[4] N. Macé, A. Jagannathan, P. Kalugin, R. Mosseri, and F. Piéchon, Phys. Rev. B 96, 045138 (2017).

[5] K. Deguchi, S. Matsukawa, N. K. Sato, T. Hattori, K. Ishida, H. Takakura, and T. Ishimasa, Nat. Mater. 11, 1013 (2012).

[6] A. Koga and H. Tsunetsugu, Phys. Rev. B 96, 214402 (2017).

[7] Y. Okabe and K. Niizeki, J. Phys. Soc. Jpn. 57, 16 (1988).

[8] A. Szallas and A. Jagannathan, Phys. Rev. B 77, 104427 (2008).

[9] S. Wessel and I. Milat, Phys. Rev. B 71, 104427 (2005).

[10] P. A. Fleury and R. Loudon, Phys. Rev. 166, 514 (1968).

[11] S. Sugai, S. Shamoto, and M. Sato, Phys. Rev. B 38, 6436 (1988).

[12] C. M. Canali and S. M. Girvin, Phys. Rev. B 45, 7127 (1992).

[13] A. V. Chubukov and D. M. Frenkel, Phys. Rev. B 52, 9760 (1995).

[14] A. W. Sandvik, S. Capponi, D. Poilblanc, and E. Dagotto, Phys. Rev. B 57, 8478 (1998).

[15] N. B. Perkins, G.-W. Chern, and W. Brenig, Phys. Rev. B 87, 174423 (2013).

[16] O. Cépas, J. O. Haerter, and C. Lhuillier, Phys. Rev. B 77, 172406 (2008).

[17] B. S. Shastry and B. I. Shraiman, Phys. Rev. Lett. 65, 1068 (1990).
[18] B. S. Shastry and B. I. Shraiman, Int. J. Mod. Phys. B 5, 365 (1991).

[19] W.-H. Ko, Z.-X. Liu, T.-K. Ng, and P. A. Lee, Phys. Rev. B 81, 024414 (2010).

[20] F. Michaud, F. Vernay, and F. Mila, Phys. Rev. B 84, 184424 (2011).

[21] Since the Hamiltonian conserves magnetization, excited states do not change the magnetization. One-magnon states $\left(|1 \mathrm{M}\rangle=\alpha_{k}^{\dagger}|0\rangle\right.$ or $\left.\beta_{l}^{\dagger}|0\rangle\right)$ belong to a different magnetization block from the ground state. Therefore, we do not need to consider the one-magnon states in our CI calculation.

[22] The $E_{1}$ representation is Raman inactive, because it does not have an in-plane second-order basis. 


\section{Graphical Table of Contents}

GTOC image:

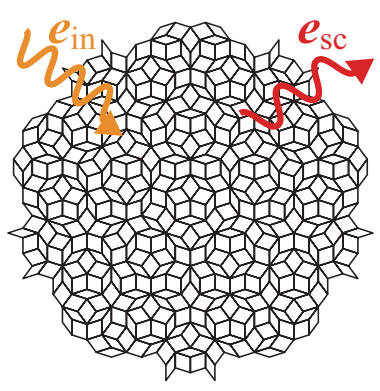

This work reports theoretical calculations of the magnetic Raman scattering on the two-dimensional quasiperiodic Heisenberg antiferromagnets. The Raman scattering intensity profile detects various magnetic excitations, including spin-chirality fluctuations. The interactions between magnons play important role in the magnetic Raman scattering process. 